livraisons

d'Histoire

de l'Architecture

\section{Livraisons de l'histoire de l'architecture}

34 | 2017

Le bois

\title{
Le renouveau de l'architecture de bois en France, 1965-1985 : une expérimentation industrielle
}

The renewal of wood architecture in France, 1965-1985

Das Wiederaufleben der Holzarchitektur in Frankreich, 1965-1985 : ein

industrielles Experimierfeld

\section{Stéphane Berthier}

\section{(2) OpenEdition}

\section{Journals}

Édition électronique

URL : http://journals.openedition.org/lha/829

DOI : 10.4000//ha.829

ISSN : 1960-5994

Éditeur

Association Livraisons d'histoire de l'architecture - LHA

Édition imprimée

Date de publication : 15 décembre 2017

Pagination : 49-60

ISSN : $1627-4970$

\section{Référence électronique}

Stéphane Berthier, « Le renouveau de l'architecture de bois en France, 1965-1985 : une

expérimentation industrielle », Livraisons de l'histoire de l'architecture [En ligne], 34 | 2017, mis en ligne le 15 décembre 2019, consulté le 22 mars 2020. URL : http://journals.openedition.org//ha/829 ; DOI :

https://doi.org/10.4000/lha.829 


\section{LE RENOUVEAU DE L'ARCHITECTURE DE BOIS EN FRANCE, 1965-1985 : UNE EXPÉRIMENTATION INDUSTRIELLE}

L'architecture de bois renaît en France dans les années 1960, après avoir été occultée au $\mathrm{XX}^{\mathrm{e}}$ siècle par les matériaux phares de la modernité tels que l'acier et le béton. À cette occasion émerge une filière industrialisée qui supplante peu à peu l'artisanat et les traditions constructives qui prévalurent dans les siècles antérieurs. Les métiers, les outils et les domaines d'application changent. Cette renaissance se fait au bénéfice des systèmes constructifs à ossature légère, descendants du balloon frame américain, contre les modes constructifs traditionnels : poteau-poutre, pans de bois, ou bois massif empilé.

De même, les formes architecturales se renouvèlent. Le bois devient un matériau moderne, l'architecture de bois rompt elle aussi avec ses traditions et se modernise. Elle échappe à l'image vernaculaire et rurale pour constituer de nouveaux imaginaires architecturaux, sensibles aux environnements naturels, à destination de populations urbaines.

Ces technologies industrielles, pour la plupart déjà ébauchées durant la première moitié du $\mathrm{XX}^{\mathrm{e}}$ siècle, vont désormais avoir une influence significative sur la production architecturale. Dans le même mouvement, cette production va servir de terrain d'expérimentation au développement de ces dispositifs techniques innovants. Architecture et technique s'y inventent réciproquement.

Ces vingt années d'innovation et de création, de 1965 à 1985, peuvent être divisées en deux périodes. La première que nous nommerons «le temps des pionniers " élabore une architecture moderne en bois sur des programmes de résidences secondaires ou de petits équipements de loisirs en plein air. Elle s'inspire de modèles architecturaux d'Amérique du Nord ou du Japon. Une seconde période, que nous appellerons « le temps du développement » fait se rencontrer, à partir du milieu des années 1970, cette nouvelle architecture de bois et la politique de l'Industrialisation Ouverte, promue par l'État français. Cette volonté publique de réorganisation de la production industrielle tirait les conclusions de l'échec des méthodes de la filière monopolistique du béton armé, par lesquelles était arrivé le désastre des "grands ensembles » à la française. L'Industrialisation Ouverte visait à conserver les avantages supposés de coût et de délais de l'industrie tout en renouant avec la diversité architecturale, pour effacer les effets appauvrissants de la standardisation. La filière bois renaissante fut un de ses terrains d'expérimentation. 


\section{Le temps des pionniers}

De nombreuses sources bibliographiques convergent pour établir la renaissance de l'architecture de bois en France au milieu des années 1960. Elles identifient trois principaux acteurs pionniers de cette expérience : les architectes Pierre Lajus, Roland Schweitzer et Jean-Pierre Watel, très impliqués dans ce mouvement. Sans doute cèdent-elles trop facilement au mythe des pères fondateurs en passant sous silence une culture constructive moderne et industrialisée qui existait déjà en France, avec une production en bois de chalets et de bungalows qui bénéficiait des méthodes élaborées durant la première moitié $\mathrm{du} \mathrm{XX}^{\mathrm{e}}$ siècle.

Ainsi, Le premier ouvrage de technologie de construction à l'ère industrielle est Holzhausbau - technik und gestaltung ${ }^{1}$ de l'allemand Konrad Wachsmann, paru en 1930, dans lequel il décline les différents modes constructifs artisanaux et détaille aussi les premiers essais d'industrialisation du balloon frame, dont la simplicité se prête bien à la standardisation. Wachsmann travaillera ensuite avec Walter Gropius, après leur immigration aux U.S.A. suite à l'arrivée du nazisme en Allemagne. À partir de 1941, ils réalisèrent ensemble le projet la Packaged House, maison en bois entièrement industrialisée, produite par la Général Panels Corporation, créée pour l'occasion.

En France, les premiers articles de revues faisant état de la construction moderne en bois remontent à 1938 avec le numéro 11 d'Architecture d'Aujourd'hui intitulé "Le bois et ses nouvelles applications dans la construction », suivi des numéros 7-8 et 9-10 de Techniques et Architecture ${ }^{3}$ de 1942. Ces trois numéros dressent un état actualisé des connaissances scientifiques et techniques du matériau bois. Ils présentent aussi les nouveaux assemblages métalliques et les bois transformés. Un panel de réalisations de grande portée illustre ces innovations présentées selon une organisation par typologie de structures. Le numéro 9-10 de Techniques et Architecture s'attache à décrire les réalisations en ossature bois dans le domaine de l'habitation. La revue présente notamment les nouveaux panneaux de contreplaqué et d'aggloméré.

Cette production industrialisée doit beaucoup aux efforts de guerre, pour la construction de baraques militaires et hébergements d'urgence ${ }^{4}$. On citera notamment le travail très approfondi que mènera Jean Prouvé avec les panneaux de bois industriels à partir de 1939 pour l'armée française, puis qu'il développera sur divers programmes jusqu'en $1966^{5}$. Cependant Jean Prouvé a construit avec le matériau bois sans pour autant produire une "architecture de bois». Il faut attendre le tournant des années 1960 pour découvrir en France une nouvelle architecture de bois,

1. Konrad Wachsmann, Holzhausbau - technik und gestaltung, Wasmuth Verlag AG, Berlin, 1930.

2. Architecture d'Aujourd'hui, n ${ }^{\circ} 11$, nov. 1938 : «Le bois et ses nouvelles applications dans la construction".

3. Techniques et Architecture, $\mathrm{n}^{\circ}$ 7-8, juil.-août et 9-10 de sept.-oct. 1942, "Le bois I et II ».

4. André Guillerme (dir.), "Le front de l'industrialisation de la construction ", Les Cahiers de la recherche architecturale et urbaine, $\mathrm{n}^{\circ} 28$, sept. 2013, p. 37-56.

5. Stéphane Berthier, "Timber in the building of Jean Prouvé: an industrial material ", Journal of Construction History Society, Cambridge, vol. 30-2, oct. 2015, p. 87-106. 
savante et reconnue par la critique, publiée dans les revues sous forme d'œuvres et exposée. Il faut aussi attendre cette période pour voir des architectes construire un discours à son sujet.

Dès 1964, Jean-Pierre Watel, jeune architecte lillois associé à l'entreprise ELCOHA présentait un prototype de maison en bois, à l'occasion de la foire de Lille ${ }^{6}$. De retour d'un voyage aux États-Unis qui l'avait convaincu que la filière de la maçonnerie produisait en France à des coûts bien supérieurs à ceux qu'il avait pu observer outre-Atlantique dans la filière bois, il eut l'idée de concevoir une maison moderne en bois, produite industriellement, pour le marché de l'habitation individuelle. Le pari de Watel était que la maîtrise industrielle de la construction bois lui permettrait d'être très compétitif. Malheureusement, seule une poignée d'exemplaires fut vendue et l'entreprise ELCOHA fit rapidement faillite. Watel explique cet échec par les réticences culturelles que le modèle suscitait à l'époque, d'abord parce que la maison préfabriquée avait mauvaise presse, ensuite parce que son architecture très moderne fut mal accueillie par un marché de la maison individuelle qui plébiscitait alors les expressions architecturales vernaculaires.

Après d'autres tentatives infructueuses Watel travaillera à partir de 1975 sur la commande d'une maison individuelle à Linselles ${ }^{7}$, pour M. Leymonerie, directeur commercial de l'entreprise Cambrai Charpente. Délaissant le système sophistiqué des panneaux industrialisés en petits bois croisés imaginé pour le prototype de la foire de Lille, Jean-Pierre Watel reviendra à la construction nord-américaine d'ossature légère à lisses verticales. Les pignons sont habillés de briques de parements tandis que les autres parties sont en bardage bois. La toiture plate composée de panneaux isolants repose sur une charpente de poutres industrielles composites à membrures en bois et diagonales métalliques.

Sur la côte aquitaine, entre 1950 et 1970, l'agence bordelaise Salier, Courtois, Lajus et Sadirac s'était fait une spécialité de la conception de belles maisons modernes, lieux de villégiature balnéaire de la bourgeoisie girondine ${ }^{8}$. Ne parvenant pas à toucher la clientèle des classes moyennes avec cette production onéreuse, l'agence décida de concevoir un modèle de maison à fabriquer en série, comme un produit concurrentiel des bungalows et chalets qui se construisaient un peu partout à cette époque. Élaborée en partenariat avec l'entreprise de charpente Guirmand, la maison Girolle 9 est une construction à l'allure vernaculaire, faite d'un ample toit débordant, à deux pans asymétriques. Son mode constructif est composé de portiques sur un entraxe de trois mètres et de cloisons porteuses en bois faite d'ossatures légères. Presque tous les éléments étaient préfabriqués en atelier et la maison pouvait être assemblée en quelques semaines. Son extrême simplicité constructive associée à ses

6. "Jean-Pierre Watel : de ses premières maisons à la maison européenne et à Village Expo ", Architecture d'Aujourd'hui, no $136,1968$.

7. Jean-Pierre Watel, "L'ossature bois, deux décennies d'expérimentations ", T\&A, n 322, 1978.

8. Michel Jacques et Annette Nève, Courtois, Salier, Lajus, Sadirac, Fouquet, atelier d'architecture Bordeaux 1950-70, Arc-en-Rêve, Bordeaux, 1997.

9. Michel Jacques et Annette Nève, op. cit., p. 42-43. 
qualités spatiales indéniables ont fait le succès d'un standard dont on estime à environ 700 le nombre d'unités construites entre 1966 et la fin des années $1970^{10}$.

En 1974, Pierre Lajus se sépara de ses associés pour monter sa propre agence. Son premier projet fut celui de sa maison à Mérignac ${ }^{11}$, entièrement en bois, qu'il termina en 1976. Ici encore, la référence à l'architecture moderne californienne est évidente. Le foyer de la maison articule plusieurs ailes qui se déploient entre les pins maritimes, sur un terrain naturel transformé à minima. La construction est préfabriquée à partir de panneaux d'ossatures légères; le chantier est alors plus un montage qu'une construction, à la manière du jeu de meccano.

À la différence de ses confrères sous influence de la modernité nord-américaine, Roland Schweitzer fut, quant à lui, inspiré par l'architecture traditionnelle japonaise. Basé à Paris, cet alsacien d'origine réalisa en 1967 un centre de vacances, à Cieux dans la Haute-Vienne ${ }^{12}$ (ill. 1).

La grande légèreté de cette construction immergée en pleine nature, les délicates relations qu'elle tisse avec la forêt, ne manquent pas d'évoquer les classiques de l'architecture japonaise. Aujourd'hui à l'abandon, cet édifice classé au patrimoine du $\mathrm{XX}^{\mathrm{e}}$ siècle, est sans doute le plus représentatif du travail de Schweitzer avec le matériau bois. Sa structure principale est une ossature poteaux-poutres régulière, sur pilotis pour ne pas modifier la topographie du terrain naturel. La toiture plate de ce bâtiment à un seul niveau lui permet de se glisser discrètement sous la frondaison des arbres. La construction ne doit rien à la charpenterie traditionnelle française mais emprunte largement à l'art japonais sans en être un pastiche. Elle en propose en effet une interprétation qui use des atouts de la modernité, avec l'emploi de connecteurs métalliques et de poutres en lamellé-collé pour les grands espaces tels que le restaurant. Les façades sont, quant à elles, réalisées en panneaux d'ossature légère à l'américaine, et habillées de lames de pin lasurées.

Ces projets pionniers ont tous été exposés en 1979 au Centre Georges Pompidou lors de l'exposition "Maisons de bois ${ }^{13}$ ». Dans l'introduction du catalogue, ce renouveau de l'architecture de bois est présenté comme : " un mouvement d'architecture rurale moderniste, inspiré des données géologiques, biologiques et climatiques du milieu ${ }^{14}$ ». Cette exposition faisait la promotion de la renaissance de la filière bois et pointait déjà les premières inflexions écologiques au sortir des Trente Glorieuses. Il y était également question de construction moderne en bois, avec les interviews de Jean Prouvé et du compagnon charpentier Ephrem Longépé qui analysaient tous deux, chacun à leur manière, la nécessité de l'industrialisation pour faire face à la demande à des plus coûts compétitifs que ceux de la filière maçonnerie ${ }^{15}$.

10. Stéphane Berthier, Entretien avec Pierre Lajus du 15 fév. 2011.

11. "Pierre Lajus, parcours d'un pionnier ", Architecture à vivre, Hors-Série, déc. 2007, p. 50-73.

12. Roland Schweitzer, Roland Schweitzer, un parcours d'architecte, Ed. Artsign, Paris, 2014, p. 198-209.

13. N. Gauzit, P. Jean, J.-P. Pigeat, R. Schweitzer, Maisons de bois, catalogue de l'exposition, Centre Georges Pompidou/CCI, 1979.

14. Maisons de bois, op. cit., p. 6-7.

15. Maisons de bois, op. cit., p. 57, 71. 


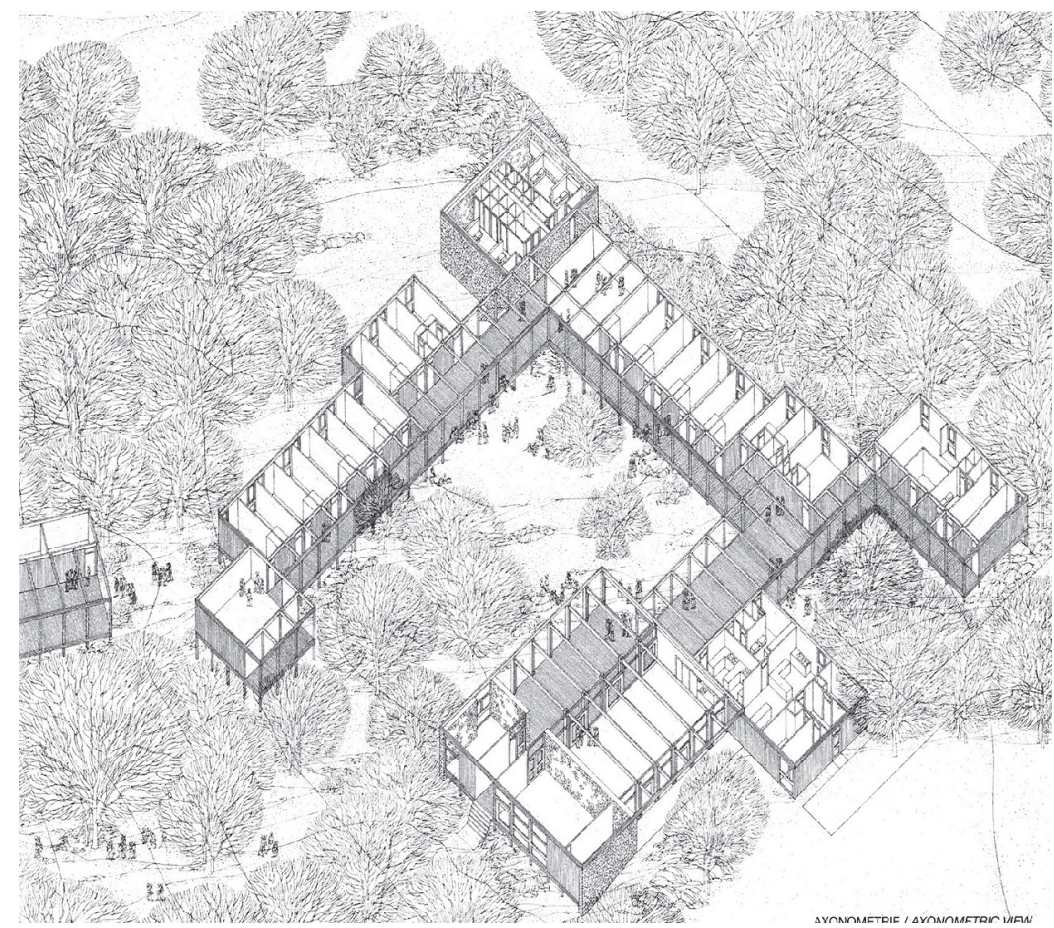

Ill. 1 : Centre de vacances à Cieux, système constructif inséré dans le site naturel, Roland Schweitzer architecte, axonométrie de l'architecte, in Roland Schweitzer, Un parcours d'architecte, Éd. Arsign, Paris, 2014, p. 199. (C) D. R.

De même, l'exposition présentait le prototype de la maison Moduli 225 imaginée en 1969 par les architectes finlandais Gullichsen et Pallasmaa, véritable kit modulaire industrialisé qui permettait une infinité de compositions possibles à partir d'une grille poteaux-poutres de 2,25 mètres par 2,25 mètres, complétée de panneaux de remplissage pleins ou vitrés ${ }^{16}$. Cette maison était présentée comme un produit de catalogue à monter soi-même. L'exposition réunissait donc un corpus d'œuvres qui renouvelait l'architecture de bois en même temps qu'un ensemble de réflexions sur la modernisation des technologies de charpente pour les concilier avec les exigences de production industrielle de l'époque.

\section{Le temps du développement}

Presque simultanément s'ouvre la seconde période de cette renaissance qui rencontre alors la politique de l'Industrialisation Ouverte. L'objectif de l'Industrialisation Ouverte était de proposer une alternative aux méthodes industrielles lourdes en vigueur depuis la reconstruction. La filière du béton armé construisait des immeubles

16. Maisons de bois, op. cit., p. 70. 
de logements surdéterminés par leur mode constructif industrialisé, et dont elle contrôlait tous les aspects. La standardisation industrielle engageant la répétitivité, la médiocrité architecturale des grands ensembles était généralement considérée comme la conséquence de ce système de production. L'administration s'inquiétait aussi de la situation de monopole de la filière béton et de ses entreprises générales qui fixaient leurs prix sans concurrence extérieure.

L'ouvrage Industrialisation ouverte, recherche et expérimentation 1971-1983 ${ }^{17}$ retrace de manière synthétique l'ensemble de ces réflexions. En 1979, la revue Techniques et architecture publie deux numéros spéciaux ${ }^{18}$ consacrés à ce sujet qui apparaît comme le grand enjeu du moment.

L'idée était de réorganiser le secteur de la construction avec, d'une part, des industriels fournissant les composants de la construction (éléments de charpente, panneaux de façade, de plancher, escaliers, équipements, etc.), comme une sorte de grand catalogue d'éléments compatibles entre eux et, d'autre part, des entreprises capables d'assembler ces composants en immeubles, à la manière d'un meccano. La dissociation entre industriels-fournisseurs et entrepreneurs-assembleurs devait rendre les coûts de construction plus transparents et augmenter les combinaisons de concurrence. De plus, ce catalogue de composants compatibles devait permettre le retour de la diversité architecturale sans perdre le bénéfice de la standardisation, mais en la ramenant à l'échelle des éléments de construction et non plus à celle des bâtiments.

Pour vérifier cette hypothèse d'industrialisation ouverte, les pouvoirs publics vont lancer en 1978 une vingtaine de réalisations expérimentales (REx), dont sept auront pour mission de tester l'hypothèse dans la filière bois ${ }^{19}$ afin de proposer une alternative au béton armé pour la construction de logements collectifs.

Assez logiquement, les architectes identifiés ci-avant vont être sollicités pour participer à ces REx. Tous ont déjà pris conscience, à travers leurs premières expériences, que les structures artisanales de la charpenterie traditionnelle n'étaient pas aptes à répondre à une demande importante à des coûts compétitifs. Ils étaient donc favorables au renouvellement de la filière grâce au développement d'unités de production industrielle, en s'appuyant sur quelques charpentiers motivés par l'innovation, tel que Cuiller en Normandie, Coquart dans le Nord ou encore Houot dans les Vosges. Dans un souci de concision, nous présenterons ici seulement les trois opérations expérimentales des architectes que nous avons présentés dans la première partie, parce qu'elles sont suffisamment caractéristiques et que les autres projets développés à cette occasion n'apportent pas d'informations supplémentaires significatives.

17. Denis Grèzes, Jean-Philippe Charon, Industrialisation ouverte, recherche et expérimentation 19711983, Paris, Ministère de l'Urbanisme et du logement, Plan Construction et Habitat, 1983, 239 p.

18. Techniques et Architecture, $\mathrm{n}^{\circ} 327$, nov. 1979 : "Industrialisation ouverte 1 : Principes et expérimentations "; $n^{\circ} 328$, janv. 1980 : "Industrialisation ouverte 2 : Systèmes constructifs et composants ".

19. Villa Morton à Bordeaux (18 logements) par Pierre Lajus ; Opération des Templiers (35 logements) à Provins par les frères Rouveau ; Quartier du Triolo à Villeneuve-d'Ascq (33 logements) par JeanPierre Watel ; Opérations Dampierre en Crot (18 logements) et Oizon (8 logements) par Christian Gimonet; La Tour du Pin (12 logements) par Denis Grèzes et Hubert Penicaud ; Opération de la rue Domrémy à Paris (29 logements) par Roland Schweitzer. 


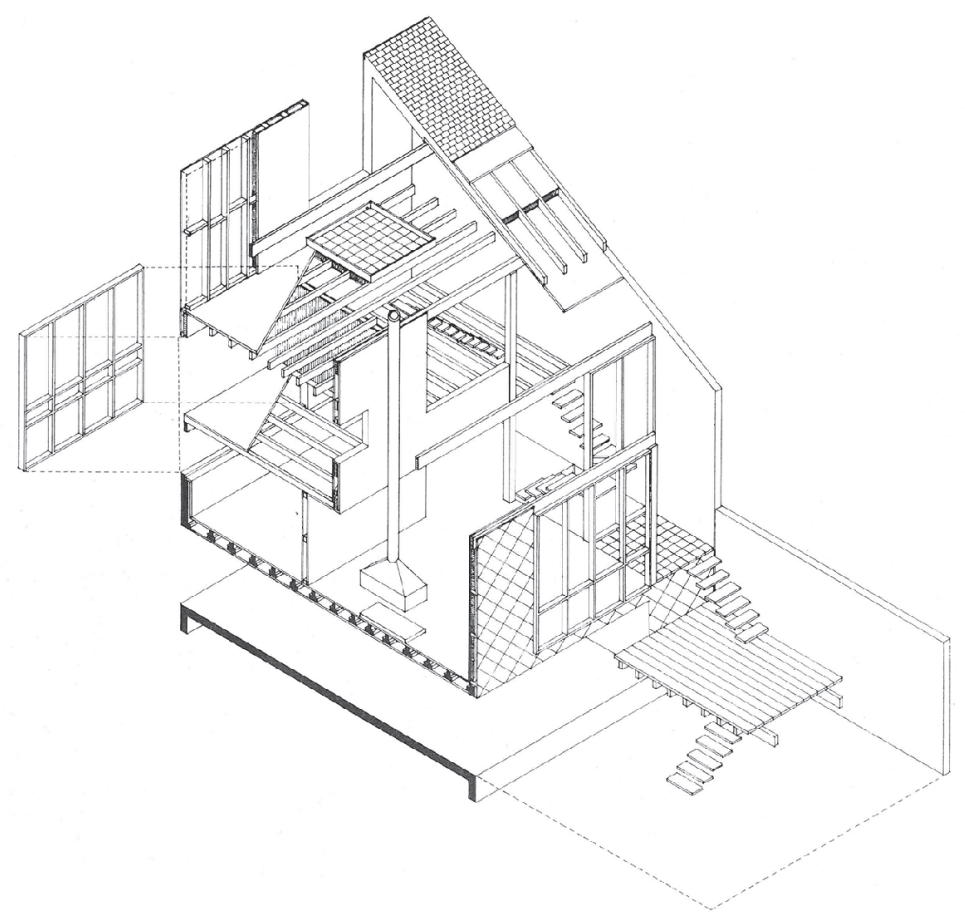

Ill. 2 : Système constructif de la villa Morton présentant les différents composants industrialisables de l'édifice, Pierre Lajus architecte, dessin axonométrique de l'architecte, Denis Grèzes et Jean-Pierre Charon, Industrialisation ouverte, recherche et expérimentation, 1971-1983, Plan Construction et Habitat, Paris, 1983, p. 178. (C) D. R.

Pierre Lajus se verra confier l'opération Morton à Bordeaux ${ }^{20}$. Cet ensemble de dix-huit logements individuels renoue avec une forme urbaine traditionnelle de maisons accolées qui forment une petite impasse perpendiculaire à la rue Morton. Un premier avant-projet publié dans $T \& A^{21}$ en 1979 présente de beaux volumes dotés de toitures monopentes élancées qui évoque le projet américain du Sea Ranch de Sonoma en Californie, construit dans les années 1960 et rapidement devenu une icône de l'architecture de bois américaine ${ }^{22}$.

L'architecte bordelais dessinait des maisons de ville, toutes différentes en fonction de leur orientation et de leur position par rapport à la rue. Mais cette diversité était réalisée à partir d'une combinatoire astucieuse d'un nombre limité de composants industriels assemblés, tels que des panneaux de façade préfabriqués, des planchers et toitures-caissons (ill. 2).

20. Denis Grèzes, Jean-Philippe Charon, op. cit., p. 170-180 ; Alix Crouzet, Les maisons à ossature bois, deux exemples en Aquitaine: la villa Morton et Villabois, 3 tomes, Mémoire de maîtrise d'Histoire de l'Art sous la dir. de M. Saboya, Université Montaigne Bordeaux III, 2004.

21. T\&A, no 327, déc. 1979, p. 114-116.

22. Gauzit, op. cit., p. 50. 


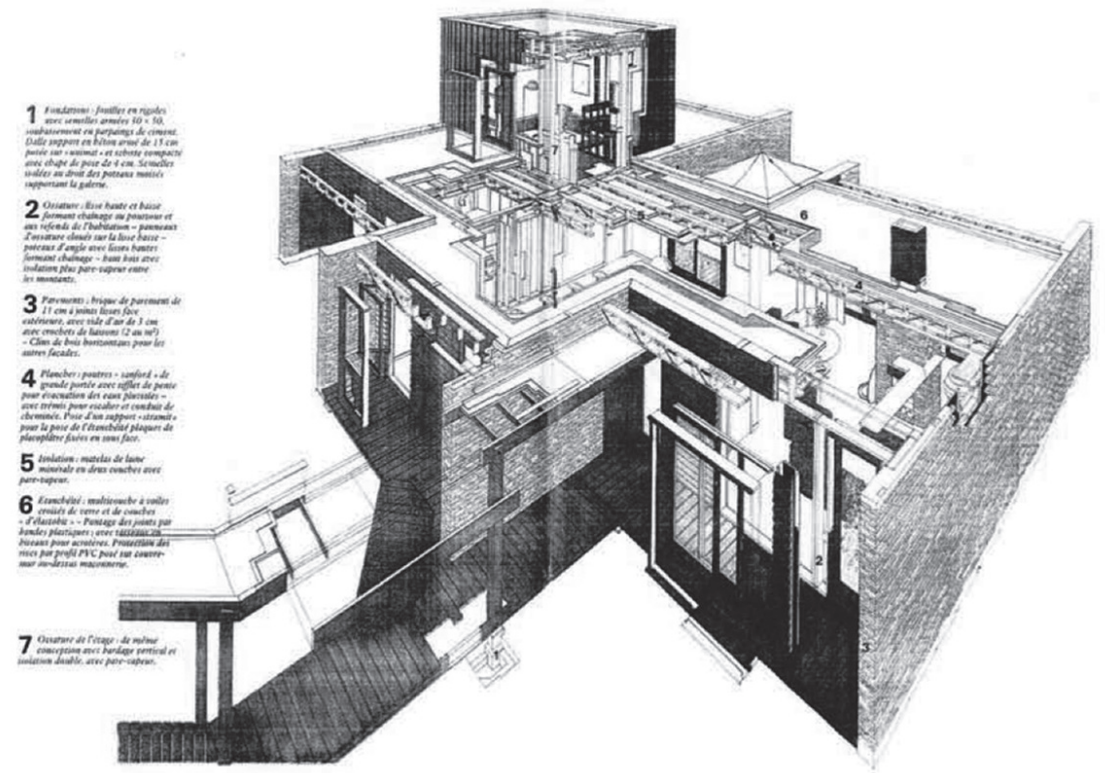

Ill. 3 : Système constructif du prototype de Linselles illustrant la construction par composants industriels, Jean-Pierre Watel architecte, axonométrie de l'architecte, in Techniques \& Architecture, n ${ }^{\circ} 327$, nov. 1979 " industrialisation ouverte I : principes et expérimentations », p. 87. (C) D. R.

L'opération réalisée montre que les ambitions architecturales et techniques initiales ont dû être revues à la baisse. L'expression architecturale s'est banalisée, les volumes se sont comprimés et les toitures sont devenues conventionnelles. Les bilans des $\mathrm{REx}^{23}$ font état de difficultés économiques avec cinq appels d'offres successifs qui ont conduit à l'abandon d'une construction par composants industriels pour revenir à des modalités de construction bois assez artisanales. Les photographies de chantier nous indiquent tout de même une part importante de préfabrication de grands éléments, mis en œuvre avec des moyens de levage modernes.

La REx de Villeneuve-d'Ascq sur laquelle travaillait Watel à la même période rencontra des difficultés similaires. Un premier projet fut publié en cours d'études dans le même numéro de $T \& A^{24}$. Il était constitué de 72 logements individuels groupés, organisés autour d'une placette publique. Les habitations étaient conçues sur la base de la maison Leymonerie que Watel rebaptisa " prototype de Linselles" et redessina à l'occasion de cette publication (ill. 3), inscrivant ainsi son travail dans une continuité de type recherche et développement $(\mathrm{R} \& \mathrm{D})^{25}$.

23. Denis Grèzes, Jean-Philippe Charon, op. cit., p. 132-146.

24. Techniques et Architecture, $\mathrm{n}^{\circ} 327$, op. cit., p. 113.

25. Stéphane Berthier, "L'appropriation de la technologie de l'ossature bois légère " in fabric $A, \mathrm{n}^{\circ} 10$, 2016, p. 13-55. 


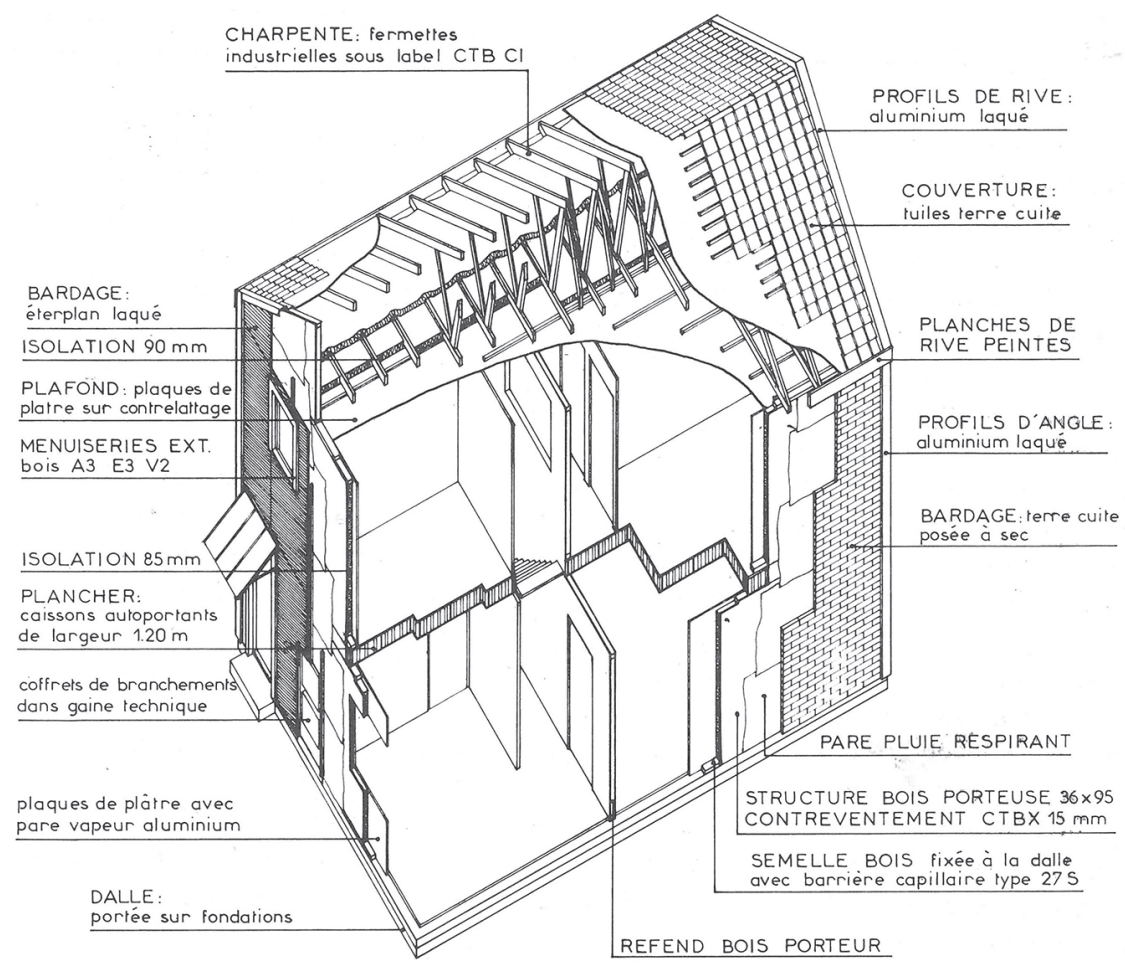

Ill. 4 : Système constructif de la réalisation expérimentale de Villeneuve-d'Ascq, Jean-Pierre Watel architecte, axonométrie de l'architecte, in Denis Grèzes, Jean-Pierre Charon, Industrialisation ouverte, recherche et expérimentation, 1971-1983, Plan Construction et Habitat, Paris, 1983, p. 139. () D. R.

Cet article exposait le principe d'une construction faite de composants industriels d'ossatures, organisés selon une règle de coordination dimensionnelle qui gérait aussi les menuiseries et le second œuvre. Toutefois, la réalisation présentée en 1983 s'avère très différente ${ }^{26}$. Elle n'est plus constituée que de 33 petites maisons compactes avec des toitures banales. Les façades y sont désormais décorées de motifs qui évoquent l'architecture vernaculaire des quartiers populaires de San Francisco plutôt que les généreuses maisons modernes de Koening, Neutra ou Elwood bâties sur les hauteurs de Los Angeles dans les années 1960. Néanmoins, ce projet mis au point avec l'entreprise Coquart est très intéressant d'un point de vue constructif $^{27}$ (ill. 4). Les façades porteuses sont réalisées avec panneaux d'ossatures légères préfabriqués, tandis que les planchers expérimentent un produit industriel composé d'un sandwich fait de panneaux de contreplaqué et d'une âme en tôle pliée.

26. Le Moniteur du 13 mai 1983, "Construire avec le bois ", p. 54-58.

27. Jean-Claude Bignon, François Lausecker, La Maison à ossature bois, fichier synoptique d'opérations, École d'Architecture de Nancy, 1983. 
Les revêtements de façades sont tous différents selon leur orientation, en clins de bois, en essentes de terre cuite, en brique du Nord ou encore en panneaux de fibrociment peints. Cette grande variété indique que l'ossature légère en bois peut être comprise comme un "porte-manteau " selon les termes de l'architecte, et recevoir n'importe quel revêtement sans lien de réciprocité entre mode constructif et expression architecturale. L'ossature bois devient un mode constructif comme un autre, banalisé, que l'on peut ou non exprimer architecturalement. Simplement, pour Watel, la filière bois et sa préfabrication en atelier est un gage de chantiers plus rapides et plus rationnels que dans la filière de la maçonnerie. Il est toutefois difficile de ne pas voir dans l'évolution des projets de Lajus et de Watel une forme de renoncement à leurs désirs d'architecture fondés sur des modèles généreux mais inadaptés à la commande de logements sociaux. Les ambitions architecturales semblent avoir disparu dans l'âpreté du dépouillement économique.

Roland Schweitzer livrera en 1983 une REx rue Domrémy à Paris ${ }^{28}$. Le bâtiment de six niveaux, sur rue, est construit avec un système mixte associant une structure porteuse en béton armé et des façades en panneaux d'ossature bois, revêtus de plaques de fibres de ciment sur les quatre premiers niveaux. Les deux niveaux d'attique sont, quant à eux, entièrement réalisés en ossature bois légère et habillés d'un bardage peint. En cour d'ilot, un petit édifice de deux niveaux accueille cinq ateliers d'artistes, construits sur le même principe que les étages d'attique.

À nouveau, ces éléments d'ossatures légères ont été préfabriqués sous formes de panneaux d'enveloppe dont les dimensions sont lisibles sur les façades de l'édifice. Simultanément à cette opération REx, Schweitzer gagnera en 1982 un concours en partenariat avec l'entreprise Houot pour la construction d'une dizaine de maisons en habitat groupé, lancé par les pouvoirs publics alsaciens, dans le but de promouvoir la filière bois locale ${ }^{29}$. Il s'agit d'un système de constructions modulaires tridimensionnelles, entièrement préfabriqués. Ces modules de $18 \mathrm{~m}^{2}$ étaient livrés entièrement équipés, au fur et à mesure de l'avancement, limitant ainsi le chantier à un simple temps d'assemblage. Malheureusement, ce concours est resté sans suite. Cette même équipe échouera encore en 1985 à réaliser soixante-deux logements sur ce même principe modulaire, à Liverdun, en Meurthe et Moselle, après la faillite du promoteur ${ }^{30}$. Cette seconde version était surmontée d'une grande toiture généreuse, elle aussi préfabriquée. Néanmoins la piste de la préfabrication tridimensionnelle était pertinente si l'on en juge aujourd'hui par le numéro 112 de la revue Séquences Bois ${ }^{31}$, intitulé "chantiers courts " qui consacre de nombreuses pages à cette technologie industrielle désormais au point.

28. Julius Natterer (dir.), Construire en bois, PPUR, Lausanne, 1987 (éd. 2007), p. 304 ; Roland Schweitzer, op. cit., p. 308-309.

29. Roland Schweitzer, op. cit., p. 334-335.

30. Roland Schweitzer, op. cit., p. 334.

31. Séquences Bois, no 112, nov. 2016. 


\section{Conclusion}

Cette renaissance de l'architecture de bois en France a été l'occasion d'inventer de nouveaux imaginaires qui témoignaient d'un désir de réconciliation avec notre environnement naturel. Mais cette renaissance fut aussi le temps de l'acculturation d'une filière professionnelle à de nouveaux modes constructifs, qui n'ont plus grandchose à voir avec la charpenterie traditionnelle. Ces nouvelles manières de faire, essentiellement nord-américaines, présentent l'avantage d'être moins sophistiquées que celles nous connaissions auparavant. En effet, la technologie de charpente nord-américaine s'est développée au XIX ${ }^{\mathrm{e}}$ siècle concomitamment à la fabrication industrielle des clous puis des vis et autres connecteurs métalliques. Ces éléments de liaison ont grandement facilité les assemblages, tandis que la charpenterie traditionnelle française, plus ancienne, est un art de l'assemblage en bois qui nécessite l'apprentissage de l'art du trait et de la taille ${ }^{32}$. D'autre part ces assemblages métalliques permettent de construire avec des petites sections, meilleur marché que les belles pièces de bois d'œuvre nécessaires à la charpente traditionnelle. La technologie américaine est donc plus économique, plus simple à mettre en œuvre et cette simplicité la rend plus facilement industrialisable. Ce mode constructif peut être préfabriqué en série, avec un outillage mécanique, sous forme de panneaux de murs, de planchers ou de toits. La lecture attentive de l'ouvrage canadien Construction de maisons à ossature bois ${ }^{33}$, édité en 1979 , nous montre que la technologie de l'ossature légère développée en France à la même période est bien similaire à celle en vigueur outre-Atlantique. Elle consiste à réaliser des constructions d'échelle modeste à partir de petites sections de bois d'environ $120 \times 50 \mathrm{~mm}$ contreventées par des panneaux de contreplaqué. Les modalités techniques sont sensiblement les mêmes des deux côtés de l'Atlantique. L'équivalence est frappante et confirme clairement qu'un transfert s'est opéré depuis l'après-guerre, de l'Amérique du Nord vers l'Europe.

À défaut d'avoir atteint les (trop) ambitieux objectifs de l'Industrialisation Ouverte, ces REx ont tout de même initié, à leur échelle, une nouvelle manière de construire en bois et jeté les bases d'une filière bois française renouvelée en développant des solutions techniques industrialisables. En 1985, le salon BatiBois de Bordeaux inaugura l'opération Villabois ${ }^{34}$ constituée de 116 maisons de bois, comme démonstrateur de la nouvelle offre de la filière régionale. L'ouvrage Habiter le bois ${ }^{35}$ publié en 1986 présente les sept REx réalisées entre 1978 et 1983, ainsi qu'une une soixantaine de réalisations de petits collectifs de logements sociaux en bois livrés de 1984 à 1986. Cette période d'une vingtaine d'années doit donc être comprise comme un temps de recherche et de développement nécessaire à l'appropriation et à l'adaptation en France d'une nouvelle technologie industrielle de construction, condition sine qua none de la crédibilité économique de la filière bois. Elle fut simultanément

32. Bernard Marrey (dir.), Histoire de bois, Éd. Picard, 1994, 199 p.

33. Coll., Construction de maisons à ossature bois-Canada, Montréal, SCHL, 1979, 375 p.

34. Crouzet, op. cit.

35. Roland Schweitzer (dir.), Habiter le bois, Ministère de l'Équipement, CSTB, 1986. 
une période de création d'une nouvelle architecture de bois, attractive et désirable, elle aussi empruntée à des modèles étrangers.

Toutefois la filière subit durement la crise économique des années 1980 et eut de grandes difficultés à poursuivre son développement hors subventions. Il ne put jamais être démontré que la construction en bois présentait un avantage économique sur la maçonnerie. Il fallut attendre le début du $\mathrm{XXI}^{\mathrm{e}}$ siècle pour que le bois trouve un second souffle avec l'émergence des exigences environnementales. Les nouveaux arguments écologiques du matériau rendent désormais acceptable le surcoût de ce mode constructif. Mais les constructions contemporaines en bois, désormais plébiscitées pour leurs vertus écologiques, sont encore réalisées avec les technologies mises au point durant cette période, certes améliorées et thermiquement plus performantes, mais toujours fondées sur le mode constructif à ossature légère industrialisée qui a remplacé les autres modes constructifs traditionnels connus antérieurement en France.

Stéphane BERTHIER architecte diplômé de l'École polytechnique fédérale de Lausanne docteur en architecture de l'université de Paris-Saclay chercheur au sein du laboratoire LéaV 